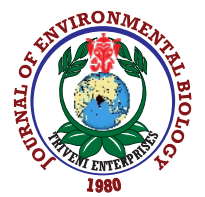

\title{
Quercetin mediated inhibition of Staphylococcus aureus biofilms and the impact of the isolate phenotype and genotype
}

\author{
E.H. Eldrehmy ${ }^{1,2}$, S.M. Abdel-Hafez ${ }^{1,3}$, Y.S. Alghamdi', M.M. Soliman ${ }^{4,5}$, S.H. Alotaibi $i^{6}$, A. Alkhedaide ${ }^{3}$, M.Y. Hassan ${ }^{1,3}$, H.H. Amer ${ }^{6,7}$ and Nada Alqadri ${ }^{1}$ \\ ${ }^{1}$ Department of Biology, Turabah University College, Taif University, 21995, SaudiArabia \\ ${ }^{2}$ Department of Microbiology, Faculty of Veterinary Medicine, Zagazig University, Zagazig, 44519, Egypt \\ ${ }^{3}$ Animal Reproduction Research Institute, Immunobiology and Immunopharmacology Unit, Giza, 11211, Egypt \\ ${ }^{4}$ Cinical Laboratories Sciences, Turabah University College, Taif University, Turabah, 21995, KSA \\ ${ }^{5}$ Biochemistry Department, Faculty of Veterinary Medicine, Benha University, Benha 13736, Egypt \\ ${ }^{6}$ Chemistry Department, Turabah University College, Turabah 29541, Taif University, Saudi Arabia \\ ${ }^{7}$ Animal Medicine and Infectious Diseases Department, Faculty of Veterinary Medicine, University of Sadat City, 32897, Egypt \\ *Corresponding Author Email : esam2005micro@gmail.com
}

\begin{abstract}
Aim: This study was designed to assess the antibiofilm activity of quercetin on characterized $S$. aureus isolates.

Methodology: This study evaluated $36 \mathrm{~S}$. aureus isolates, each of which was identified using Gram staining, culture, biochemical, and PCR assays. Isolates were cultured and their biofilm production was evaluated using Congo red agar (CRA) plates, microtiter plate tests and PCR, and the effects of quercetin were examined.

Results: The CRA results revealed that eight (22.3\%) S. aureus isolates were strongly positive for biofilm production and an additional 18 isolates (50\%) showed moderate biofilm capacity. The remaining 10 isolates were negative $(27.7 \%)$ for biofilm production. S. aureus isolates were divided into strong positive, intermediate, and negative groups, $27.8 \%, 44.5 \%$, and $27.7 \%$, respectively. Scanning electron microscopy showed that the biofilm-producing isolates appeared as aggregates of cells within a heavy matrix. In addition, PCR assay identified IcaA and IcaD (66.6\% for both) biofilm production genes in most isolates and IcaC (61.1\%), IcaB, FnbB (33.3\% for both), and Fib (22.2\%) in several other strains. Quercetin significantly inhibited biofilm activity in biofilm producing $S$. aureus isolates in a dose-dependent manner, with an inhibition rate of $29.6-87.7 \%$.

Interpretation: Biofilm production is dependent on Ica gene phenotype and strains with an IcaABCD or IcaABD phenotype produce more biofilm than strains with

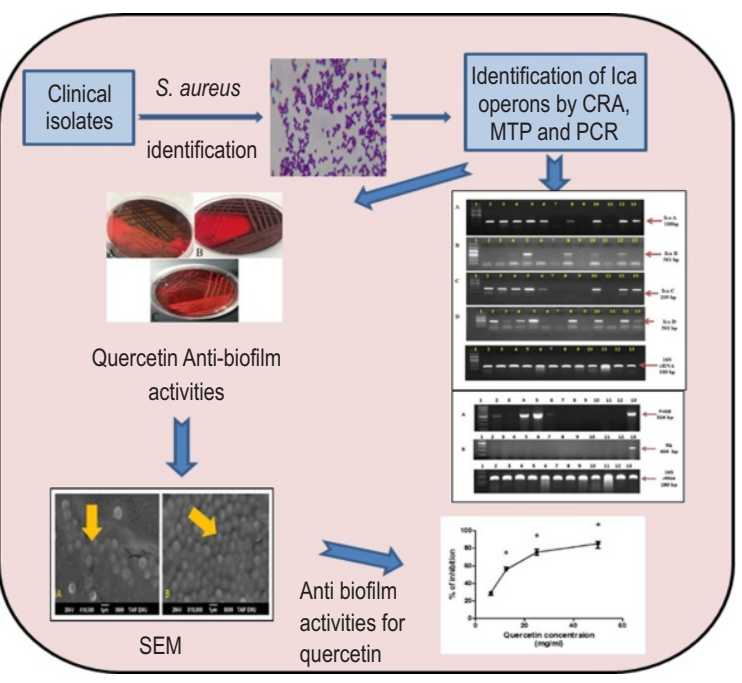
IcaAD phenotype. Quercetin significantly inhibited $S$. aureus biofilm production, irrespective of Ica phenotype.

Key words: Biofilm, Congo red agar, Ica operon, PCR, Quercetin, S. aureus

How to cite : Eldrehmy, E.H., S.M. Abdel-Hafez, Y.S. Alghamdi, M.M. Soliman, S.H. Alotaibi, A. Alkhedaide, M.Y. Hassan, H.H. Amer and Nada Alqadri: Quercetin mediated inhibition of Staphylococcus aureus biofilms and the impact of the isolate phenotype and genotype. J. Environ. Biol., 42, 615-624 (2021). 


\section{Introduction}

Staphylococcus spp. are common skin microbes known to inhabit the human skin, sweat glands, and mucous membranes, especially in the nasal cavity of healthy individuals (Costa et al., 2011; Plata et al., 2009). S. aureus is also present as a commensal in several other niches inside human body and can act as an opportunistic pathogen with its aberrant growth linked to infection of skin and soft tissues. These infections can even lead to more severe diseases such asosteomyelitis, endocarditis, pneumonia, septicemia and toxic shock (Lowy, 1998; Peacock and Paterson, 2015; Rao et al., 2015). Moreover, S. aureus has been implicated in food poisoning and scalded skin syndrome (Haasnoot and De Vries, 2018; Vitale et al., 2015). Several environmental bacteria have also been implicated in both severe acute and chronic infections in humans, with the severity and complexity of these diseases largely dependent on their ability to produce multilayered cellular matrices, known as biofilms (Tsuneda et al., 2003). Biofilms are layers of bacteria within a glycocalyx composed of polysaccharides, DNA and proteins. These films are also implicated in increasing resistance to antibiotics and immune defenses (Chung and Toh, 2014).

Phenotypic identification of biofilm-producing S. aureus strains can be completed using Congo red agar, microtiter plate assay and tube methods. Recently, polymerase chain reaction has also been used to assay for bacterial surface components, which may encode adhesive matrix molecules (MSCRAMMs) and Ica operon (Gerke et al., 1998). Biofilm production is dependent on two properties: adherence of bacterial cells to the surface facilitating the production of multi-layered cell clusters (Ghasemian et al., 2015) and biosynthesis of polysaccharide intercellular adhesion (PIA) compounds (Ikonomidis et al, 2009). These intercellular adhesion (Ica) molecules are produced from four open reading frames (ORFs), IcaA, IcaB IcaC, and IcaD (Cucarella et al., 2002), which encode the proteins needed to synthesize the adhesion molecules and are followed by the IcaR gene which acts as irregulator (Arciola et al., 2001). IcaR protein negatively regulates the expression of ICaADBC by binding to the promoter and restricting transcription (Parsek and Fuqua, 2004). This repression can be released by various other regulatory factors including SarA and stress-induced sigma factor SigB which act in a positive feedback loop to increase biofilm synthesis (Beenken et al., 2003; Jefferson et al., 2003).

Autoinducer 2 (Al-2) is also known to control this system and has been previously described as a universal language for interspecies communication, especially in $S$. aureus. Al-2 precursor molecule regulates rbf transcription and decreases PIA-dependent biofilm production in S. aureus (Ma et al., 2017). Ica operons were first reported in S. epidermidis (Heinrichs et al., 1996) and then in S. aureus (Cramton et al., 1999). S. aureus attaches to various surface materials, including host tissues and surgical devices, which is mediated by its ability to produce a variety of adhesion molecules in addition to those produced by the Ica operons (Beenken et al., 2004). Most adhesion molecules recognize glycoproteins found in the extracellular matrix and plasma of patients. Diversity of these adhesion molecules can be attributed to the ability of $S$. aureus to adapt to a wide range of growth environments, including connective tissues, bone, blood stream and vascular tissues. These surface-uncovered proteins are recognized and communicated within $S$. aureus communities via microbial surface components recognizing adhesive matrix molecules (MSCRAMMs), which are capable of initial attachment to native tissues and medical devices (Christensen et al., 1985).

Inhibition of these biofilms has obvious clinical benefits, highlighting the need to develop novel therapeutics targeting these structures. Given this, it is not surprising that this remains a focus of research for many international groups and has given rise to a wide variety of anti-biofilm compounds with unique structures, including herbal compounds, chelating agents, antibiotics, antimicrobial peptides and synthetic chemicals (Sadekuzzaman et al., 2015). Quercetin is a plant-derived flavonol present in several food products, including capers, onions, peppers, cranberries, tomatoes, apples, and grapes (Nabavi and Silva, 2018). Quercetin has a documented antibiofilm effect against several Gram-positive and Gramnegative bacterias (Earl et al., 2008). Quercetin affect anti-biofilm activities by decreasing the total protein and viable cells within the biofilm (Zeng et al., 2019). Thus, the present study examined and summarized the phenotypic and genotypic isolation and characterization of $S$. aureus biofilms. Moreover, the possible treatment of biofilms formed by these isolates was also checked.

\section{Materials and Methods}

Isolation of clinical samples: $S$. aureus was isolated from infected patients. A total of 36 samples were collected from King Faisal Hospital, Taif, Saudi Arabia. These samples were isolated from wound swabs (28/36), blood (4/36), sputum (2/36) and catheter (2/36) and collected between September 2019 and February 2020.

Identification of isolates: All isolates were initially identified using conventional bacterial identification tests such as Gram stain, catalase, coagulase and mannitol salt agar specific for $S$. aureus (Kot et al., 2018). Isolates were stored at $-20^{\circ} \mathrm{C}$ for further study.

Congo Red Agar method (CRA): Bacterial isolates were cultured following the method of (Freeman et al., 1989) with minor modifications. The medium consisted of brain heart infusion broth (BHI) supplemented with $1 \%$ glucose. Congo red was set up as an independent aqueous solution and autoclaved before it was added to agar plates, inoculated and incubated at $37^{\circ} \mathrm{C}$ for $24 \mathrm{hr}$. Strong biofilm producers showed intense black colonies with dry crystalline consistency, while intermediate producers created colonies with darkened center or black colonies without a dry crystalline consistency. Pink colonies indicated weak slime CRA.

Microtiter plate assays: Adherence assays on microtiter plates were used as the in-vitro measure of biofilm activity as described 
by (Cafiso et al., 2007) with minor modification. Briefly, S. aureus isolates ( 36 strains) were inoculated in TSB media supplemented with $0.5 \%$ glucose and incubated at $37^{\circ} \mathrm{C}$ for $18 \mathrm{hr}$. After that, a

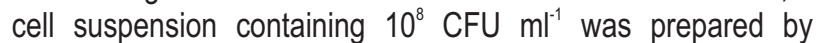
transferring the incubated culture to new tryptic soy broth with $0.5 \%$ glucose. A $200 \mu \mathrm{l}$ of this cell suspension was then loaded onto a 96 well microtiter plate in triplicate and incubated for $48 \mathrm{hr}$ at $37^{\circ} \mathrm{C}$ to evaluate biofilm productions. The plates were then washed twice with sterile phosphate-buffered saline (PBS) and fixed in $250 \mu \mathrm{l}$ of methanol for $15 \mathrm{~min}$. The plates were then removed and stained with crystal violet $(1 \%, 200 \mu \mathrm{l})$ for $5 \mathrm{~min}$, rinsed with running tap water and then air-dried. The colorant was dissolved in $95 \%$ ethanol to measure absorbance at $492 \mathrm{~nm}$ and any values of $\geq 0.12$, were considered positive for biofilm production. Any samples producing values of $<0.2$ were regarded as weak biofilm producers, 0.2-0.4 as moderate producers and $>0.4$ as strong producers, respectively.

Scanning electron microscope study of produced biofilm: Biofilms were visualized using SEM following incubation on glass slides $(1 \mathrm{~cm} \times 1 \mathrm{~cm})$. Plates were incubated at $37^{\circ} \mathrm{C}$ for $48 \mathrm{hr}$ and then, the glass slides were washed twice with PBS (PH7.4) and fixed at $4^{\circ} \mathrm{C}$ for $2 \mathrm{hr}$ in $3 \%$ glutaraldehyde. The samples were dehydrated in an ethanol gradient $(30 \%, 50 \%, 70 \%, 90 \%$, and $100 \%, 10$ min each) and then dried and sputter-coated with gold before being examined under a high-resolution scanning microscope (Priester et al., 2007). These biofilms were photographed under SEMatthe Electron Microscope Unit of Taif University (model JEOLJSM-6390 LA serial number PM14400099).

DNA extraction of S. aureus strains: $S$. aureus colonies were purified and cultivated in $1 \mathrm{ml}$ of tryptic soy broth for $24 \mathrm{hr}$ at $37^{\circ} \mathrm{C}$. DNA was then extracted from 50-100 bacterial colonies suspended in $400 \mu \mathrm{IDEPC}$ water and boiled for $10 \mathrm{~min}$ at $100^{\circ} \mathrm{C}$. The tubes were then centrifuged at $14000 \mathrm{rpm}$ for $7 \mathrm{~min}$ and a clear supernatant was used for PCR amplification after measuring the concentration of DNA on a BIO-RAD spectrophotometer.

PCR amplification: Specific primers were used to facilitate species specific identification of the Ica genes (Table 1). These primers were designed using a TaqMan primer designer program and the primers were purchased from Macrogen (GAsa-dong, Geumcheon-gu. Korea). PCR was performed in a final volume of $25 \mu \mathrm{l}$ and consisted of $5 \mu \mathrm{l}$ DNA template, $1 \mu \mathrm{l}$ of $10 \mathrm{pM}$ forward and reverse primers, and $12.5 \mu \mathrm{l}$ master mix, and the volume was adjusted with sterilized deionized water. PCR was then performed using the following cycle conditions: $94^{\circ} \mathrm{C}$ for $5 \mathrm{~min}$, followed by 35 cycles of denaturation at $94^{\circ} \mathrm{C}$ for $60 \mathrm{sec}$, annealing as described in Table 1 , and extension at $72^{\circ} \mathrm{C}$ for 60 $\mathrm{sec}$, with a final extension for $7 \mathrm{~min}$ at $72^{\circ} \mathrm{C}$. 16S rRNA gene was used as an amplification control and PCR products were visualized using a $2 \%$ agarose gel stained with ethidium bromide in Tris-Borate-EDTA (TBE) buffe rand UV light and photographed using a gel documentation system (SynGen, USA).
Quercetin antibiofilm activity: To assess the efficiency of quercetin mediated biofilm inhibition, strains were treated with various doses of quercetin, $50,25,12.5$, and $6.25 \mathrm{mg} \mathrm{ml}^{-1}$ in DMSO. Quercetin was purchased from Sigma-Aldrich (St. Louis, MO, USA) and biofilm inhibition was assayed as follows. Mature biofilms were established by inoculating wells with heavy

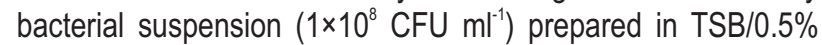
glucose, and then aerobically incubated with shaking at $37^{\circ} \mathrm{C}$ for $48 \mathrm{hrs}$. The media were discarded, and the wells were washed with sterile PBS ( $\mathrm{pH} 7.2)$. These biofilms were then treated with various concentrations of quercetin under aerobic conditions at $37^{\circ} \mathrm{C}$ for $24 \mathrm{hrs}$ and then stained and quantified as described above. All tests were performed in triplicate for three different experiments.

Statistical analysis: Data obtained from the study were expressed as mean \pm S.E. for all 36 S. aureus isolates. Data were analyzed using analysis of variance (ANOVA) with Bonferroni test in SPSS software version 11.5, for Windows (SPSS, IBM, Chicago, IL, USA) and statistical significance was set at $P<0.05$.

\section{Results and Discussion}

CRA assay (Table 2) revealed that eight (22.3\%) isolates showing intense black color were strongly positive for the production of biofilms, while 18 other isolates (50\%) were intermediate producers and the remaining 10 isolates (27.7\%) were negative for biofilm production (Fig.1). In addition, these results showed that isolates from sputum tended to be the strongest biofilm producers (100\%), with blood $(25 \%)$ and wound swab isolates $(17.8 \%)$ demonstrating a much smaller capacity for biofilm production. In contrast, none of the catheter isolates were biofilm producers (100\%). Quantitative MPT was then conducted based on the CRA test results. After reading the absorbance at $492 \mathrm{~nm}$, the strains were classified as strong, moderate and weak biofilm producers.

The results of all 36 human clinical $S$. aureus isolates were identified as $10(27.7 \%)$ strong biofilm producers, 16 $(44.4 \%)$ intermediate producer strains and $10(27.7 \%$, Table 2$)$ non-producer strains. S. aureus, which is commonly found in the nasal cavity of healthy individuals (30\%), is both a genetic carrier for and producer of biofilms (Kluytmans and Wertheim, 2005). $S$. aureus biofilm production is enhanced by increasing glucose concentrations up to $0.5 \%$ in TSB media (O'Neill et al., 2007). Biofilm-producing isolates were detected using CRA containing $0.5 \%$ glucose and this assay is the most reproducible, fastest and easiest initial indicator of biofilm production currently available, relying on a simple color based determination of activity (Arciola et al., 2002). The results of CRA test identified more positive isolates than MTP assay which is consistent with the previous data (Gowrishankar et al., 2016b). Of all the S. aureus strains examined, eight showed a clear strong positive phenotype $(22.2 \%)$ for biofilm production and these results are similar to the previous reports where $88.9 \%$ of $S$. aureus isolates analyzed produced some form of biofilm (Ammendolia et al., 1999). It is well 
Table1: Sequence details of PCR primers used to detectthe Ica genes in the PCR assays

\begin{tabular}{|c|c|c|c|c|}
\hline Target Gene & Annealing conditions & Amplicon size & Strand & Sequence(5'-3') \\
\hline IcaA & $50^{\circ} \mathrm{C}, 45 \mathrm{sec}$ & $188 \mathrm{bp}$ & $\begin{array}{l}\text { Sense } \\
\text { Antisense }\end{array}$ & $\begin{array}{l}\text { CAACCTCAACTAACGAAAGGTAG } \\
\text { GTCTAAGAAGTTTGCTGTTATG }\end{array}$ \\
\hline$I c a B$ & $48^{\circ} \mathrm{C}, 45 \mathrm{sec}$ & $561 \mathrm{bp}$ & $\begin{array}{l}\text { Sense } \\
\text { Antisense }\end{array}$ & $\begin{array}{l}\text { GTGTTAGTCAATCACAATTTGAATC } \\
\text { CATTGGAGTTCGGAGTGACTG }\end{array}$ \\
\hline IcaC & $50^{\circ} \mathrm{C}, 45 \mathrm{sec}$ & $209 b p$ & $\begin{array}{l}\text { Sense } \\
\text { Antisense }\end{array}$ & $\begin{array}{l}\text { GTCACAGTTACTGACAACCTTG } \\
\text { CAATGAGTCTAGAATGATTGGATG }\end{array}$ \\
\hline$I c a D$ & $50^{\circ} \mathrm{C}, 45 \mathrm{sec}$ & $501 \mathrm{bp}$ & $\begin{array}{l}\text { Sense } \\
\text { Antisense }\end{array}$ & $\begin{array}{l}\text { GTTGGTATCCGACAGTATACTG } \\
\text { CGTGAATCGTCATCTGCATTTG }\end{array}$ \\
\hline fib & $55^{\circ} \mathrm{C}, 60 \mathrm{sec}$ & $404 \mathrm{bp}$ & $\begin{array}{l}\text { Sense } \\
\text { Antisense }\end{array}$ & $\begin{array}{l}\text { CTACAACTACAATTGCCGTCAACAG } \\
\text { GCTCTTGTAAGACCATTTTCTTCAC }\end{array}$ \\
\hline$F n b B$ & $55^{\circ} \mathrm{C}, 60 \mathrm{sec}$ & $524 b p$ & $\begin{array}{l}\text { Sense } \\
\text { Antisense }\end{array}$ & $\begin{array}{l}\text { GTAACAGCTAATGGTCGAATTGATACT } \\
\text { CAAGTTCGATAGGAGTACTATGTTC }\end{array}$ \\
\hline $16 S \mathrm{rRNA}$ & $50^{\circ} \mathrm{C}, 45 \mathrm{sec}$ & $180 \mathrm{bp}$ & $\begin{array}{l}\text { Sense } \\
\text { Antisense }\end{array}$ & $\begin{array}{l}\text { GTTGGGCAGTCTAAGTTGACT } \\
\text { CTTCATGTAGTCGAGTTGCAG }\end{array}$ \\
\hline
\end{tabular}

Table 2: Distribution of Ica genes in biofilm producing isolates identified using Congo red agar (CRT) and Microtitre plate test (MPT) assays

\begin{tabular}{llllllll}
\hline Isolate origin & $\begin{array}{l}\text { No. of } \\
\text { samples }\end{array}$ & SPI N & CRA & & SPIN & MPT \\
\hline Wound swab & 28 & $5 / 28$ & $15 / 28$ & 8 & $6 / 28$ & $14 / 28$ & $8 / 28$ \\
Catheter & 2 & - & - & 2 & - & - & 2 \\
Blood & 4 & $1 / 4$ & $3 / 4$ & - & $2 / 4$ & $2 / 4$ & - \\
Sputum & 2 & 2 & - & - & $2 / 2$ & - & - \\
Total samples & 36 & $22.2 \%$ & $50 \%$ & $27.7 \%$ & $27.7 \%$ & $44.4 \%$ & $27.7 \%$ \\
\hline
\end{tabular}

Values represent experiments completed in triplicate. SP: strong positive; I: intermediate; N: negative; CRA: Congo red agar and MPT: microtitre plate test.

established that the microtiter plate test is a more quantitative and accurate test for biofilm detection in $S$. aureus with high accuracy, specificity and positive predictive values (Mathur et al., 2006). The microtiter plate test results of 36 S. aureus isolates identified in this study revealed 26 isolates as biofilm producers, 10 of which were strong producers $(27.7 \%), 16$ were intermediate (44.4\%), and 10 produced nobiofilms, which is in agreement with the results of CRA assay. SEM was used to visualize these biofilms on glass slides and the presence of heavy clump biofilms, composed of aggregates of bacterial cells embedded in a matrix, confirmed strong biofilm producer label applied to several $S$. aureus isolates of this study (arrowheads in Fig. 2B).

The negative biofilm producers appeared round with the characteristic grape shape without a matrix (Fig. 2). The SEM results also confirmed $S$. aureus aggregation in response to biofilm production confirming our earlier assays. Biofilm production is dependent on the expression of polysaccharide intercellular adhesion (PIA) compounds (Mack et al., 1996) and previous reports have linked the presence of $I c a A D B C$ genes with PIA production and the occurrence of biofilms as genes in this operon mediate intercellular adherence and production of multilayer biofilms (Lappin-Scott and Bass, 2001). Given this data, further study was conducted to determine the prevalence of

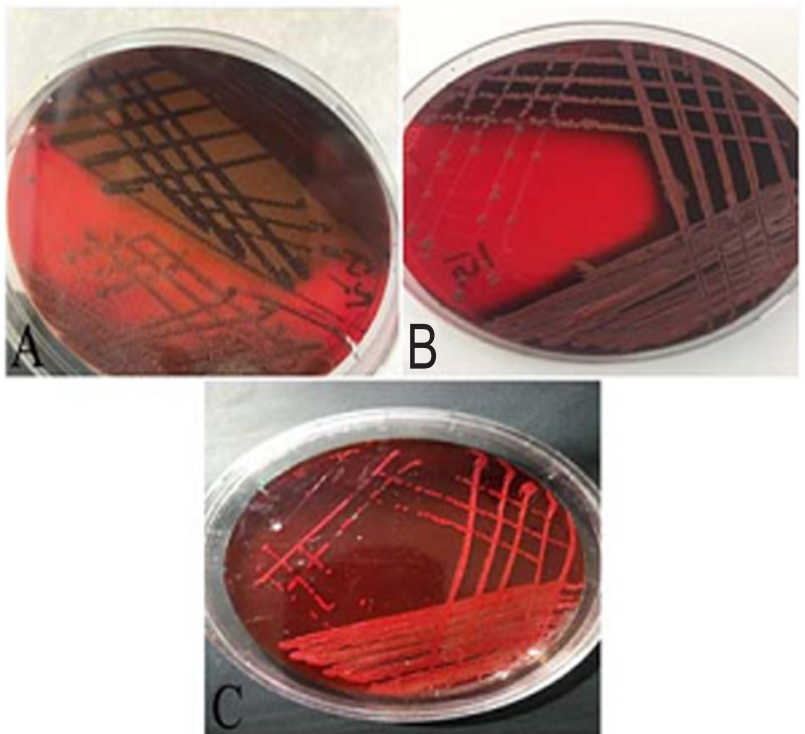

Fig. 1: Congo red agar based detection of $S$. aureus biofilm producers: (A) Black colonies with dry crystalline consistency (strong positive); (B) Dark colonies without the dry crystalline phenotype and blackening of centers (intermediate producers) and (C) Smooth pink colonies (none producers). 

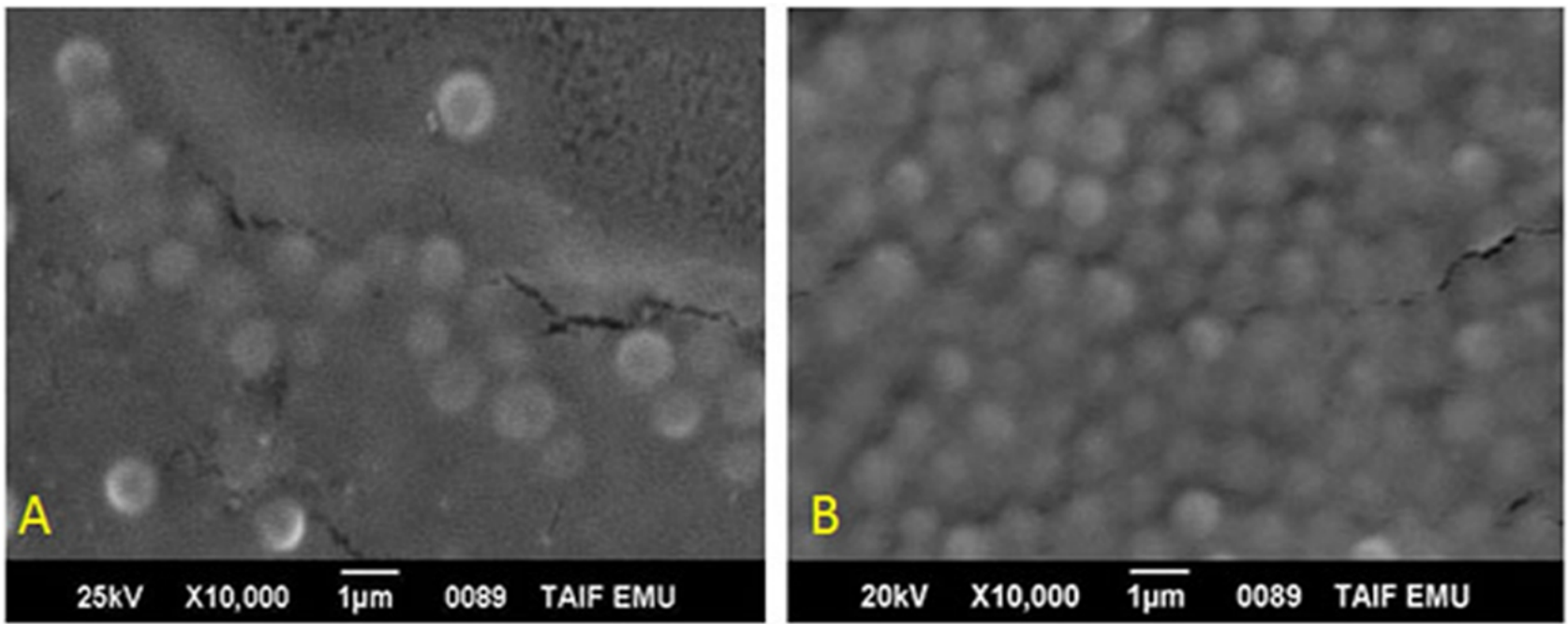

Fig. 2: SEM micrographs showing: (A) biofilm negative S. aureus isolates (Grape-like cells without matrix) and (B) biofilm producing $S$. aureus isolates (Cell aggregates embedded in a matrix).
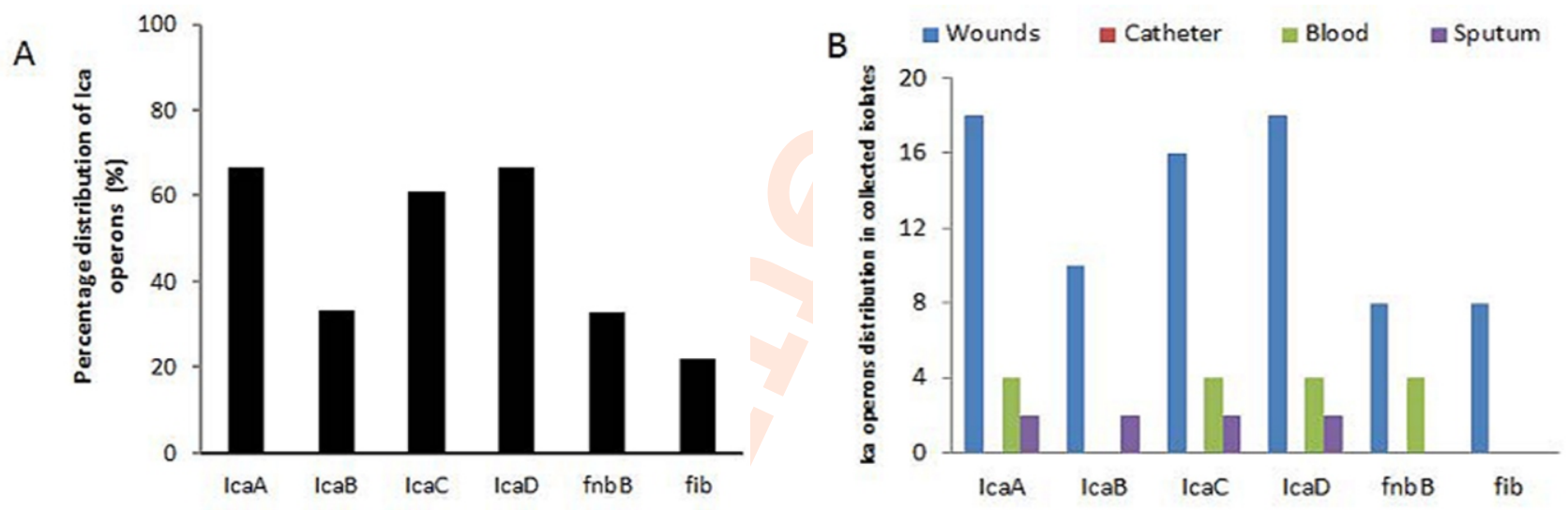

Fig. 3: (A) Percentage of samples positive for IcaABCD operon genes: IcaA and $I c a D: 66 \%, I c a C, 61.1 \%, I c a B$ and fnbB, 33.3\% and fib, $22.2 \%$; (B) Distribution of $I c a A B C D$ positive samples. All data are expressed as mean from three different experiments.

Ica operon in these isolates using PCR against the IcaA (188 bp), IcaB (561 bp), IcaC (209 bp), IcaD (501 bp), FibA (404bp) and FnbB (524bp) genes using 16S rRNA gene as a control (180 bp). Analysis of 36 isolates revealed that $I c a A$ and $I c a D$ were present in 24 isolates $(66.6 \%)$, and their distribution was abundant in samples isolated from sputum, blood and wound swabs (Fig. 3A$\mathrm{B}$; Fig. 4). The IcaC gene was detected in 22 isolates (61.1\%) distributed evenly between the sputum, blood, and wound swabs (Fig. 4), while IcaB and FnbB were detected in 12 (33.3\%) samples of sputum and wound swabs (Fig. 4,5A).

Finally, the fib gene was only detected in 8 isolates (22.2\%) (Fig. 5 B). PCR data also revealed that five out of 36 isolates did not harbor any IcaADBC operons (13.8\%), which was consistent with the results of Gowrishankar et al. (2016a). It is worth noting that all of the strong biofilm-producing strains harbored both $I c a A D B C$ and $I c a A D B$. IcaD was also present in both the intermediate and weak biofilm-producing isolates. Our data is in agreement with other studies that have shown that all strong biofilm-producing $S$. aureus isolates contain IcaABCD and $I c a A B D$, while IcaAD is more common in weak to moderate producers (Piechota et al., 2018). The IcaA protein plays a crucial role in the synthesis of PIA, while $I c a D$ does not possess any exclusive transferase activity (Gupta et al., 2017).

Current data suggests that the expression of $I c a A$ is associated with massive biofilm production. Distribution of IcaA among the present isolates studied was $66.6 \%$ and was 


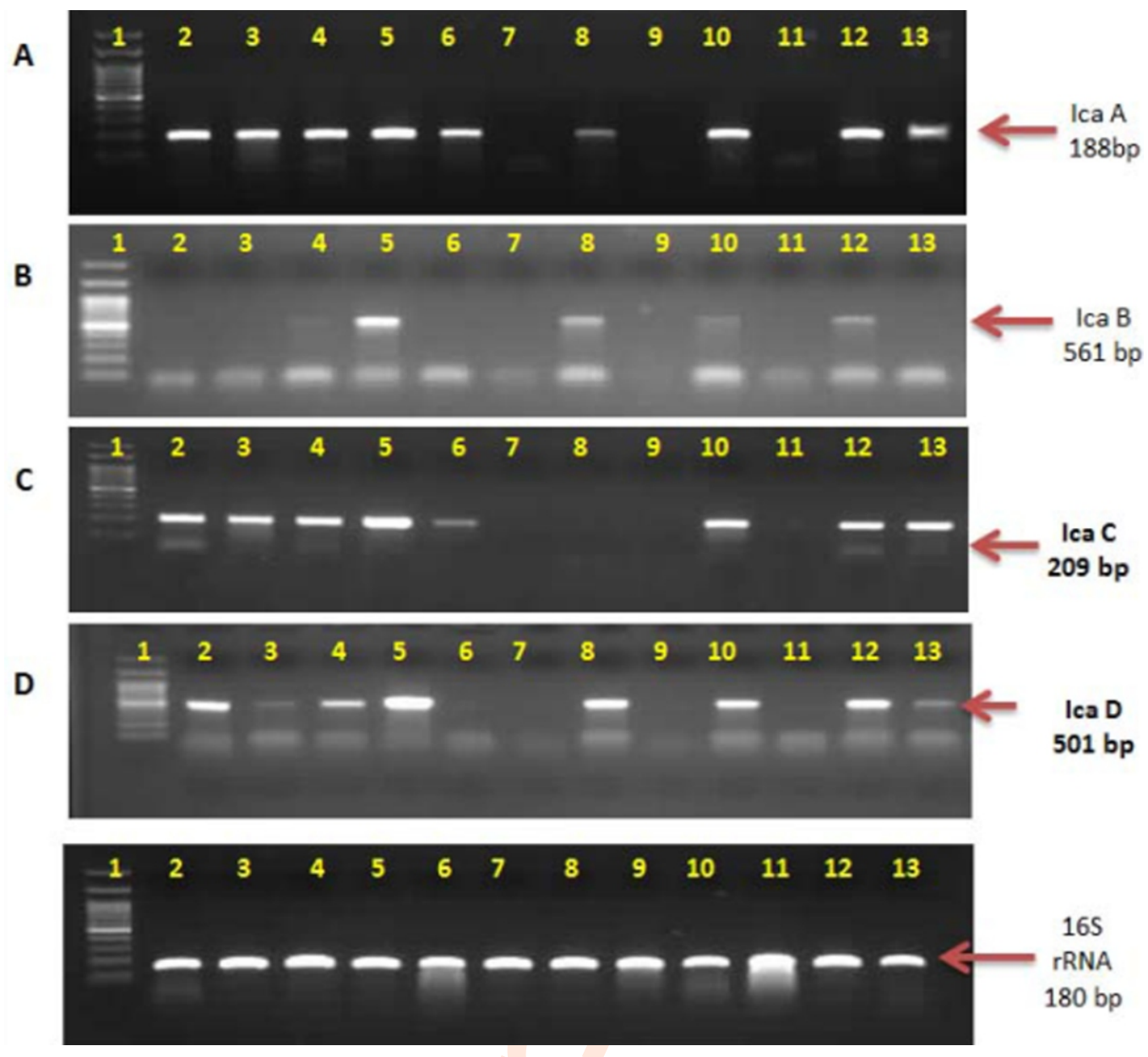

Fig. 4: Amplification of Ica $A, B, C$ and $D$ genes from $S$. aureus isolates using species specific PCR. Upper panel describes the visualization of these results; lane 1: 100 bp DNA ladder and lanes 2-13 are identified $S$. aureus isolates. Lower panel shows amplification of $16 S$ rRNA gene in these sample samples.

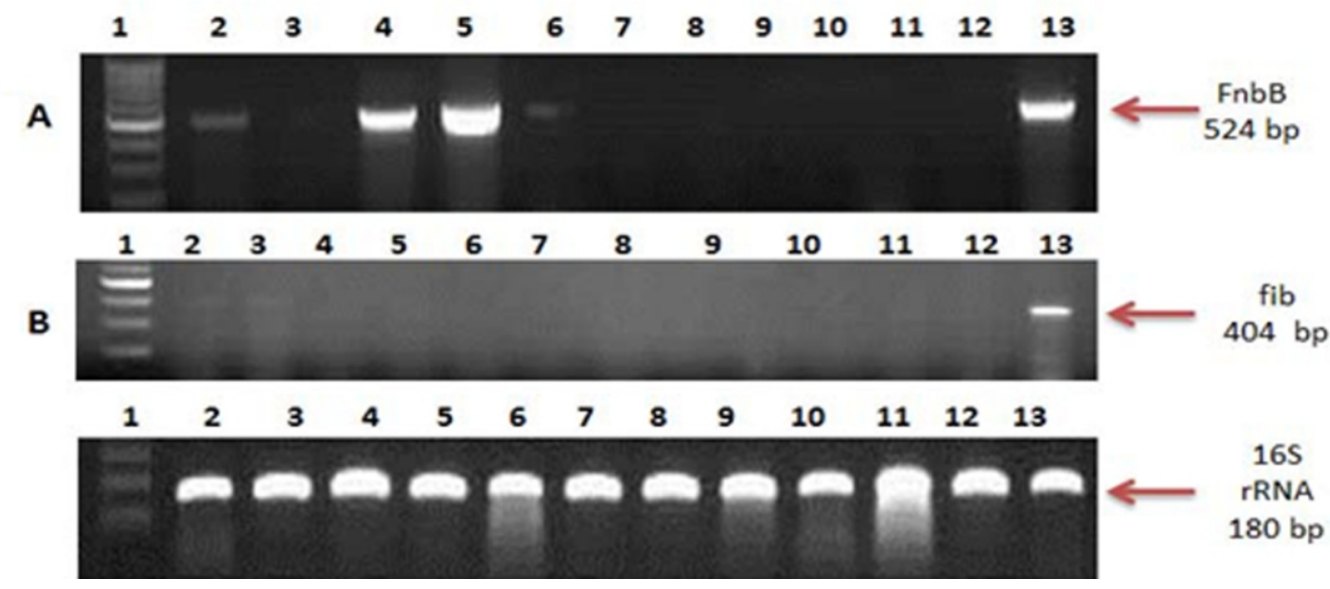

Fig. 5: Amplification of FnbB and fib genes in S. aureus isolates using species specific PCR. Upper panel shows target gene amplification in the S. aureus isolates; lane 1 is $100 \mathrm{bp}$ DNAladder and lanes 2-13 are S. aureus isolates. Lower panel shows $16 \mathrm{~S}$ rRNAamplification in these samples. 


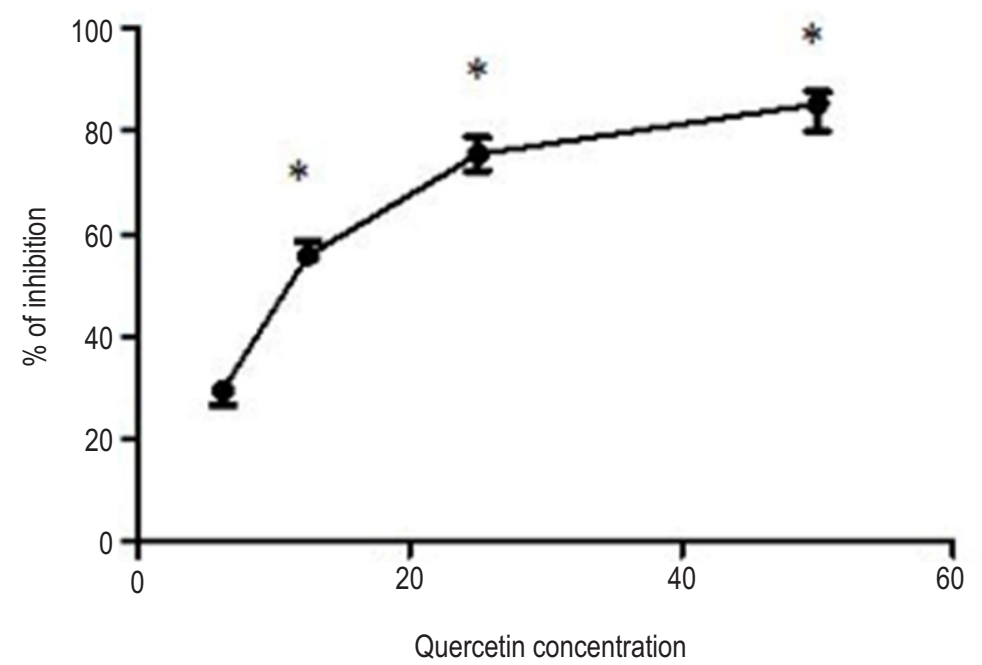

Fig. 6: Anti- biofilm effect of quercetin against $S$. aureus isolates. Maximum effected was achieved using $50 \mathrm{mg} \mathrm{m}^{-1}$ which totally inhibited biofilm production, reducing it by $87.7 \%$.

expressed in all of our strong biofilm-producing strains. Other previous studies (de Silva et al., 2002; Ninin et al., 2006) described the relationship between the presence of IcaAD operon and massive biofilm production, while others did not find this association, which is consistent with our findings. Another study reported that the prevalence of $I c a A$ and $I c a D$ was $66 \%$ and $58.4 \%$, in S. aureus isolates (Kroning et al., 2016), which was also consistent with our results. IcaB possess deacetylase, which deacetylates poly- $\mathrm{N}$-acetylglucosamine. Deacetylation is critical for biofilm formation and has been linked to biofilm development in MRSA MSSA. MRSA MSSA biofilm production has also been shown to be IcaADBC dependent and responsive to specific environmental signals (Pokrovskaya et al., 2013). Furthermore, the absence of $I c a B$ leads to a defect in the synthesis of poly-Nacetyl glucosamine and less efficient binding to bacterial cell surface decreasing biofilm production (Vuong et al., 2004).

IcaC is an integral membrane protein that may convey $\mathrm{N}$ acetylglucosamine oligomers across the bacterial cell membrane. IcaB deacetylates PIA activity (Gotz, 2002). This study demonstrated $61.1 \%$ prevalence of IcaC. Conversely, clfA and $F n b B(A$ and $B$ ) adhesions are important in host cell binding and encode MSCRAMMs, required for adhesion to abiotic surfaces. Hypothetically, fibronectin-binding proteins (FnbA and $F n b B$ ) act to facilitate invasion by modulating the adhesion and internalization of bacterial cells into the host tissues. In addition, it is well established that fibronectin proteins facilitate primary adherence and intercellular accumulation in biofilms increasing colony aggregation (Heilmann, 2011). However, it was found that only $33.3 \%$ and $22.2 \%$ of the test isolates were positive for the FnbB and fib genes, respectively (Table 2). Interestingly, the prevalence of $F n b B$ and fib were $17.9 \%$ and $71.8 \%$ in their isolates (Patel et al., 2009), however, variation in these results may be correlated to variation of bacterial strains in different geographical regions. Grouping of biofilm-producing S. aureus was demonstrated by others who grouped $S$. aureus isolates into three clusters based on their potential biofilm production creating a blood isolates, colonizing intravenous devices, and commensal isolates from the skin or nose categories (Agarwal and Jain, 2013). Our findings clarify that the strongest biofilm-producing strains were isolated from sputum, blood and wound swabs, with prevalence rates of $100 \%, 50 \%$, and $21 \%$, respectively, which may be due to difficulty of host respiratory environment, which causes the bacteria to produce intensive biofilm to resist these adverse conditions. Moreover, blood isolates need to invade blood vessels which may be aided by the secretion of adhesion genes and increased biofilm production. Quercetin had good bacteriostatic effect on S. aureus and E. coli (Wang et al., 2021). Incubating these biofilms with different concentrations of quercetin resulted in significant inhibition of their biofilm production in a dose-dependent manner (Fig. 6).

Recently, several flavonoids, including quercetin have been identified as strong contenders for the production of antibiofilm agents (Matilla-Cuenca et al., 2020; Memariani et al., 2019). Quercetin was able to eradicate pre-prepared $S$. aureus biofilms at $50 \mathrm{mg} \mathrm{ml}^{-1}$, which completely inhibited biofilm production (87.7\%) in a dose-dependent manner. A contemporary study demonstrated the inhibitory effect of quercetin on both clinical and reference isolates of $S$. aureus (250-500 $\mathrm{g} \mathrm{m} \mathrm{ml}^{-1}$ ) (da Costa Júnior et al., 2018) and another study reported that quercetin crippled biofilm production in $S$. aureus isolates when applied at various concentrations (1-50 $\mathrm{mg} \mathrm{m}^{-1}$ ) (Kim et al., 2018) which is consistent with our findings. Comparable results show that quercetin also demonstrated reliable antibiofilm activity against $S$. mutans, which may be used for treating dental caries (Zeng et al., 2019). 
S. aureus biofilm production differs according to sample type with sputum isolates being the strongest biofilm producers and wound swab isolates the weakest biofilm producers. All strains positive for IcaABCD and IcaABD genes were potent biofilm producers, producing significantly more biofilms than strains positive only for IcaAD. Quercetin significantly inhibited biofilm production and showed good ameliorative effects. Quercetin may be useful in the eradication of biofilm-forming $S$. aureus isolates, which may persist in the hospital environment potentially increasing antibiotic resistance in these environments.

\section{Acknowledgments}

We greatly appreciate the contributions of all authors and the Deans of Scientific Research Affairs at Taif University, Saudi Arabia for their financial support of this project via grant number \# 6064-439-1 financed by Taif University.

\section{Add-on Information}

Authors' contribution: E.H. Eldrehmy: Designed the experiment and carried out the phenotypic characterization of the bacterial isolates; S.M. Abdel-Hafez: carried out the phenotypic characterization of bacterial isolates; Y.S. Alghamdi: Interpreted the data and prepared the manuscript; M.M. Soliman: Carried out PCR assays and revised the paper; S.H. Alotaibi: interpreted data and prepared manuscript; A. Alkhedaide: Carried out PCR assays and revised the paper; M.Y. Hassan: Examined the antibiofilm activity of quercetin; H.H. Amer: Interpreted the data and prepared the manuscript; Nada Alqadri: carried out the phenotypic characterization of the bacterial isolates.

Research content: The research content of manuscript is original and has not been published elsewhere.

Ethical approval: The Scientific Research Ethical Committee of Turabah University College, Taif University, Saudi Arabia signed off all procedures used in this study. The Scientific Deanship of Taif University, Saudi Arabia, along with its Ethical Committee, approved all procedures used in this study for the project \#6064439-1.

Conflict of interest: The authors declare that there is no conflict of interest.

\section{Data from other sources: NotApplicable}

Consent to publish: All authors agree to publish the paper in Journal of Environmental Biology.

\section{References}

Agarwal, A. and A. Jain: Glucose and Sodium chloride induced biofilm production and ica operon in clinical isolates of staphylococci. Indian J. Med. Res., 138, 262-266 (2013).

Ammendolia, M., R.D. Rosa, L. Montanaro, C. Arciola and L. Baldassarri: Slime production and expression of the slime-associated antigen by staphylococcal clinical isolates, J. Clin. Microbiol., 37, 3235-
3238 (1999).

Arciola, C.R., L. Baldassarri and L. Montanaro: Presence of icaA and icaD genes and slime production in a collection of staphylococcal strains from catheter-associated infections. J. Clin. Microbiol., 39, 2151-2156 (2001).

Arciola, C.R., D. Campoccia, S. Gamberini, M. Cervellati, E. Donati and L. Montanaro: Detection of slime production by means of an optimised Congo red agar plate test based on a colourimetric scale in Staphylococcus epidermidis clinical isolates genotyped for ica locus. Biomaterials, 23, 4233-9 (2002).

Beenken, K.E., J.S. Blevins and M.S. Smeltzer: Mutation of sarA in Staphylococcus aureus limits biofilm formation. Infect. Immun., 71, 4206-42011 (2003).

Beenken, K.E., P.M. Dunman, F. McAleese, D. Macapagal, E. Murphy, S.J. Projan, J.S. Blevins and M.S. Smeltzer: Global gene expression in Staphylococcus aureus biofilms. J. Bacteriol., 186, 4665-4684 (2004)

Cafiso, V., T. Bertuccio, M. Santagati, V. Demelio, D. Spina, G. Nicoletti and S. Stefani: Agr-Genotyping and transcriptional analysis of biofilm-producing Staphylococcus aureus. FEMS Immunol. Med. Microbiol., 51, 220-227 (2007).

Christensen, G.D., W.A. Simpson, J.J. Younger, L.M. Baddour, F.F. Barrett, D.M. Melton and E.H. Beachey: Adherence of coagulasenegative staphylococci to plastic tissue culture plates: A quantitative model for the adherence of staphylococci to medical devices. J. Clin. Microbiol., 22, 996-1006 (1985).

Chung, P.Y. and Y.S. Toh: Anti-biofilm agents: Recent breakthrough against multi-drug resistant Staphylococcus aureus. Pathog. Dis., 70,231-239 (2014)

Costa, S.S., C. Falcao, M. Viveiros, D. Machado, M. Martins, J. MeloCristino, L. Amaral and I. Couto: Exploring the contribution of efflux on the resistance to fluoroquinolones in clinical isolates of Staphylococcus aureus. BMC Microbiol., 11, 241 (2011).

Cramton, S.E., C. Gerke, N.F. Schnell, W.W. Nichols and F. Gotz: The intercellular adhesion (ica) locus is present in Staphylococcus aureus and is required for biofilm formation. Infect. Immun., 67, 5427-5433 (1999).

Cucarella, C., M.A. Tormo, E. Knecht, B. Amorena, I. Lasa, T.J. Foster, and J.R. Penades: Expression of the biofilm-associated protein interferes with host protein receptors of Staphylococcus aureus and alters the infective process. Infect. Immun., 70, 3180-3186 (2002).

da Costa Júnior, S.D., J.V. de Oliveira Santos, L.A. de Almeida Campos, M.A. Pereira, N.S.S. Magalhães and I.M.F. Cavalcanti: Antibacterial and antibiofilm activities of quercetin against clinical isolates of Staphyloccocus aureus and Staphylococcus saprophyticus with resistance profile. Int. J. Environ. Agricul. Biotechnol., 3, 266213 (2018).

de Silva, G.D., M. Kantzanou, A. Justice, R.C. Massey, A.R. Wilkinson, N.P. Day and S.J. Peacock: The ica operon and biofilm production in coagulase-negative Staphylococci associated with carriage and disease in a neonatal intensive care unit. J. Clin. Microbiol., 40, 382-388 (2002).

Earl, A. M., R. Losick and R. Kolter: Ecology and genomics of Bacillus subtilis. Trends Microbiol., 16, 269-275 (2008).

Freeman, D.J., F.R. Falkiner and C.T. Keane: New method for detecting slime production by coagulase negative staphylococci. J. Clin. Pathol., 42, 872-874 (1989)

Gerke, C., A. Kraft, R. Sussmuth, O. Schweitzer and F. Gotz: Characterization of the $\mathrm{N}$-acetylglucosaminyltransferase activity involved in the biosynthesis of the Staphylococcus epidermidis polysaccharide intercellular adhesin. J. Biol. Chem., 273, 18586- 
$18593(1998)$

Ghasemian, A., S.N. Peerayeh, B. Bakhshi and M. Mirzaee: The microbial surface components recognizing adhesive matrix molecules (MSCRAMMs) genes among clinical isolates of Staphylococcus aureus from hospitalized children. Iran J. Pathol., 10, 258-264 (2015).

Gotz, F.: Staphylococcus and biofilms. Mol. Microbiol., 43, 1367-78 (2002).

Gowrishankar, S., A. Kamaladevi, K. Balamurugan and S. K. Pandian: Invitro and in-vivo biofilm characterization of methicillin-resistant Staphylococcus aureus from patients associated with pharyngitis infection. Biomed. Res. Int., 2016, 1289157 (2016a).

Gowrishankar, S., A. Kamaladevi, K. Balamurugan and S. K. Pandian: Invitro and in-vivo biofilm characterization of methicillin-resistant Staphylococcus aureus from patients associated with pharyngitis infection, Biomed. Res. Int., 2016, 1289157 (2016b).

Gupta, A., S. Mishra, S. Singh and S. Mishra: Prevention of IcaA regulated poly $\mathrm{N}$-acetyl glucosamine formation in Staphylococcus aureus biofilm through new-drug like inhibitors: In silico approach and MD simulation study. Microb. Pathog., 110, 659-669 (2017).

Haasnoot, P.J. and A. De Vries: Staphylococcal scalded skin syndrome in a 4-year-old child: A case report. J. Med. Case Rep., 12, 20 (2018).

Heilmann, C.: Adhesion mechanisms of staphylococci. Adv. Exp. Med. Biol., 715, 105-23 (2011).

Heinrichs, J.H., M.G. Bayer and A.L. Cheung: Characterization of the sar locus and its interaction with agr in Staphylococcus aureus. J. Bacteriol., 178, 418-423 (1996).

Ikonomidis, A., A. Vasdeki, I. Kristo, A. N. Maniatis, A. Tsakris, K. N. Malizos and S. Pournaras: Association of biofilm formation and methicillin-resistance with accessory gene regulator (agr) loci in Greek Staphylococcus aureus clones. Microb. Pathog., 47, 341344 (2009)

Jefferson, K.K., S.E. Cramton, F. Gotz and G.B. Pier: Identification of a 5nucleotide sequence that controls expression of the ica locus in Staphylococcus aureus and characterization of the DNA-binding properties of IcaR. Mol. Microbiol., 48, 889-899 (2003).

Kim, M.K., T.G. Lee, M. Jung, K.H. Park and Y. Chong: In-vitro synergism and anti-biofilm activity of quercetin-pivaloxymethyl conjugate against Staphylococcus aureus and Enterococcus species. Chem Pharm. Bull. (Tokyo), 66, 1019-1022 (2018).

Kluytmans, J.A. and H.F. Wertheim: Nasal carriage of Staphylococcus aureus and prevention of nosocomial infections. Infection, 33, 3-8 (2005).

Kot, B., K. Wierzchowska, A. Gruzewska and D. Lohinau: The effects of selected phytochemicals on biofilm formed by five methicillinresistant Staphylococcus aureus. Nat. Prod. Res., 32, 1299-1302 (2018).

Kroning, I.S., M.A. Iglesias, C.P. Sehn, T.K. Valente Gandra, M.M. Mata and W.P. da Silva: Staphylococcus aureus isolated from handmade sweets: Biofilm formation, enterotoxigenicity and antimicrobial resistance. Food Microbiol., 58, 105-111 (2016).

Lappin-Scott, H.M. and C. Bass: Biofilm formation: Attachment, growth, and detachment of microbes from surfaces. Am. J. Infect. Cont., 29, 250-251 (2001).

Lowy, F. D.: Staphylococcus aureus infections. N. Engl. J. Med., 339, 520-532(1998).

Ma, R., S. Qiu, Q. Jiang, H. Sun, T. Xue, G. Cai and B. Sun: Al-2 quorum sensing negatively regulates rbf expression and biofilm formation in Staphylococcus aureus. Int. J. Medi. Microbiol., 307, 257-267 (2017).

Mack, D., W. Fischer, A. Krokotsch, K. Leopold, R. Hartmann, H. Egge and R. Laufs: The intercellular adhesin involved in biofilm accumulation of Staphylococcus epidermidis is a linear beta-1,6linked glucosaminoglycan: purification and structural analysis. J. Bacteriol., 178, 175-83 (1996).

Mathur, T., S. Singhal, S. Khan, D.J. Upadhyay, T. Fatma and A. Rattan: Detection of biofilm formation among the clinical isolates of Staphylococci: An evaluation of three different screening methods. Indian J. Med. Microbiol., 24, 25-29 (2006).

Matilla-Cuenca, L., C. Gil, S. Cuesta, B. Rapún-Araiz, M. Žiemytè, A. Mira, I. Lasa and J. Valle: Antibiofilm activity of flavonoids on staphylococcal biofilms through targeting BAP amyloids. Sci. Rep., 10, 18968 (2020).

Memariani, H., M. Memariani and A.Ghasemian: An overview on antibiofilm properties of quercetin against bacterial pathogens. World J. Microbiol. Biotechnol., 35, 143 (2019).

Nabavi, S.M. and A.S. Silva: Nonvitamin and nonmineral nutritional supplements. $1^{\text {st }}$ Edn., Academic Press. 583 pages (2018).

Ninin, E., N. Caroff, E. Espaze, J. Maraillac, D. Lepelletier, N. Milpied and H. Richet: Assessment of ica operon carriage and biofilm production in Staphylococcus epidermidis isolates causing bacteraemia in bone marrow transplant recipients. Clin. Microbiol. Infect., 12, 446-452 (2006).

O'Neill, E., C. Pozzi, P. Houston, D. Smyth, H. Humphreys, D.A. Robinson and J.P. O'Gara: Association between methicillin susceptibility and biofilm regulation in Staphylococcus aureus isolates from device-related infections. J. Clin. Microbiol., 45, 1379-1388 (2007).

Parsek, M.R. and C. Fuqua: Biofilms 2003: emerging themes and challenges in studies of surface-associated microbial life. $\mathrm{J}$. Bacteriol., 186, 4427-4440 (2004).

Patel, J.B., R.J. Gorwitz and J.A. Jernigan: Mupirocin resistance. Clin Infect. Dis., 49, 935-941 (2009).

Peacock, S.J. and G.K. Paterson: Mechanisms of methicillin resistance in Staphylococcus aureus. Annu. Rev. Biochem., 84, 577-601 (2015).

Piechota, M., B. Kot, A. Frankowska-Maciejewska, A. Gruzewska and A. Wozniak-Kosek: Biofilm formation by methicillin-resistant and methicillin-sensitive Staphylococcus aureus strains from hospitalized patients in Poland. Biomed. Res. Int., 2018, 4657396 (2018).

Plata, K., A.E. Rosato and G. Wegrzyn: Staphylococcus aureus as an infectious agent: Overview of biochemistry and molecular genetics of its pathogenicity. Acta. Biochim. Pol., 56, 597-612 (2009).

Pokrovskaya, V., J. Poloczek, D.J. Little, H. Griffiths, P.L. Howell and M. Nitz: Functional characterization of Staphylococcus epidermidis IcaB, a de-N-acetylase important for biofilm formation. Biochemistry, 52, 5463-5471 (2013).

Priester, J.H., A.M. Horst, L.C. Van de Werfhorst, J.L. Saleta, L.A. Mertes and P.A. Holden: Enhanced visualization of microbial biofilms by staining and environmental scanning electron microscopy. J. Microbiol. Methods., 68, 577-587(2007).

Rao, Q., W. Shang, X. Hu and X. Rao: Staphylococcus aureus ST121: A globally disseminated hypervirulent clone. J. Med. Microbiol., 64, 1462-1473 (2015).

Sadekuzzaman, M., S. Yang, M.F.R. Mizan and S.D. Ha: Current and recent advanced strategies for combating biofilms. Comprehen. Revi. Food Sci. Food Saf., 14, 491-509 (2015).

Tsuneda, S., H. Aikawa, H. Hayashi, A. Yuasa and A. Hirata: Extracellular polymeric substances responsible for bacterial adhesion onto solid surface. FEMS Microbiol. Lett., 223, 287-292 (2003).

Vitale, M., M.L. Scatassa, C. Cardamone, G. Oliveri, C. Piraino, R. Alduina and C. Napoli: Staphylococcal food poisoning case and 
molecular analysis of toxin genes in Staphylococcus aureus strains isolated from food in Sicily, Italy. Foodborne Pathog. Dis., 12, 21-23 (2015).

Vuong, C., S. Kocianova, J.M. Voyich, Y. Yao, E.R. Fischer, F.R. DeLeo and M. Otto: A crucial role for exopolysaccharide modification in bacterial biofilm formation, immune evasion and virulence. J. Biol. Chem., 279, 54881-6 (2004).
Wang, Z., W. Zou, L. Liu, M. Wang, F. Li and W. Shen: Characterization and bacteriostatic effects of $\beta$-cyclodextrin/quercetin inclusion compound nanofilms prepared by electrospinning. Food Chem., 338, 127980 (2021).

Zeng, Y., A. Nikitkova, H. Abdelsalam, J. Li and J. Xiao: Activity of quercetin and kaemferol against Streptococcus mutans biofilm. Arch. Oral. Biol., 98, 9-16 (2019). 